\title{
Template-based synthesis and discontinuous hysteresis loops of cobalt nanotube arrays
}

\author{
Huimin Zhang $\cdot$ Xiuli Zhang • Tianshan Wu • \\ Zhijun Zhang $\cdot$ Jinfei Zheng $\cdot$ Huiyuan Sun
}

Received: 6 April 2013/Accepted: 25 June 2013/Published online: 4 July 2013

(C) The Author(s) 2013. This article is published with open access at Springerlink.com

\begin{abstract}
Co nanotubes were successfully prepared in the pores of anodic aluminium oxide templates using a DC electrodeposition method. The dependence of the product morphology on the applied potential was studied over the range -1.0 to $-3.0 \mathrm{~V}$. The results showed that the wall thickness of the nanotubes became thinner as the applied potential was reduced, and that there existed a critical potential $\left(v_{\mathrm{c}}\right)$ related to the electrodeposition parameters, below or above which the electrodeposition process is dominated by kinetics and thermodynamics, respectively. The formation of nanotubes is the result of kinetics dominating the electrodeposition process. Magnetic measurements showed that the hysteresis loops of the nanotubes were discontinuous. Theoretical analysis suggested the existence of some peculiar magnetization configuration such as a vertical Bloch line, which was responsible for the discontinuity of the hysteresis loops.
\end{abstract}

H. Zhang $\cdot$ X. Zhang $\cdot$ T. Wu $\cdot$ Z. Zhang $\cdot$ J. Zheng $\cdot$

H. Sun $(\bowtie)$

College of Physics Science and Information Engineering,

Hebei Normal University, Shijiazhuang 050024, China

e-mail: huiyuansun@126.com

H. Zhang

e-mail: zhanghuimin73101@ 163.com

H. Zhang

College of Materials Science and Engineering, Hebei University

of Science and Technology, Shijiazhuang 050018, China

H. Zhang $\cdot$ X. Zhang $\cdot$ T. Wu $\cdot$ Z. Zhang $\cdot$ J. Zheng $\cdot$ H. Sun

Key Laboratory of Advanced Films of Hebei Province,

No. 20 Road East of 2nd Ring South, Yuhua District,

Shijiazhuang 050024, Hebei, China

\section{Introduction}

An exciting research subject in material science is the fabrication and investigation of nanostructures such as nanowires and nanotubes because of their unique properties and potential applications in various areas, such as catalysis [1], sensing [2], field emission [3], hybrid solar cells and electronic devices $[4,5]$. Ferromagnetic nanotubes have drawn special attention in recent years because their magnetic anisotropy and magnetic reversal mechanism are much different from those of nanowires [6-10]. Ferromagnetic Co nanotubes have single axis magnetic anisotropy, which makes them a promising material for future applications in magnetic sensors and ultrahigh density magnetic recording. The synthesis of Co nanotubes has focused on anodic aluminium oxide (AAO) template-based electrodeposition because it is not only a convenient inexpensive technique, but also modification of the AAO template is not necessary for the fabrication of nanotubes, which eliminates the introduction of impurities in the nanotubes [11]. To obtain nanotubes, the common method used in template-based electrodeposition is to control the applied current densities to a level higher than $20 \mathrm{~mA} / \mathrm{cm}^{2}$. However, this often results in a lower filling rate and inhomogeneous tube length $[12,13]$. In this paper, we report the fabrication of Co nanotube arrays embedded in AAO templates with a high filling rate and uniform growth. The dependence of Co morphology on the applied potential has also been studied. A critical potential $\left(v_{\mathrm{c}}\right)$ for obtaining Co nanotube by template-based electrodeposition method is represented. The experimental results show that the formation of Co nanotubes is the result of kinetics dominating the electrodeposition process. Magnetic measurements were carried out using a physical property measurement system (PPMS) at room temperature. The 
results showed that hysteresis loops of the Co nanotubes are discontinuous. A possible reason is discussed. This work will benefit understanding of the electrochemical growth mechanism of metal nanotubes as well as the magnetization reversal of ferromagnetic nanotubes.

\section{Experimental}

We fabricated anodic oxide templates as follows: in order to eliminate internal stress, a high-purity (99.999\%) aluminium (Al) foil was thermally treated at $400{ }^{\circ} \mathrm{C}$ for $2 \mathrm{~h}$ and then brought to room temperature. The whole process is in an argon atmosphere to prevent the $\mathrm{Al}$ from oxidation. Next, the heat-treated Al foil was electropolished in a mixed solution of alcohol and perchloric acid (ratio by volume 4:1) for $5 \mathrm{~min}$ to smooth the surface. To obtain highly ordered pores, a two-step anodization was used. In the first step, the $\mathrm{Al}$ foil was anodized at $5{ }^{\circ} \mathrm{C}$ and $50 \mathrm{~V}$ DC in $0.3 \mathrm{M}$ oxalic acid for about $8 \mathrm{~h}$. The resulting aluminium oxide layer was then removed by immersing the anodized $\mathrm{Al}$ in a mixed solution of $0.4 \mathrm{M}$ chromic acid and $0.6 \mathrm{M}$ phosphoric acid (ratio by volume 1:1) at room temperature. Subsequently, the samples were reanodized under the same anodization conditions as in the first step. This resulted in the formation of AAO nanopores with diameters of $50 \mathrm{~nm}$. After the second anodization, the remaining $\mathrm{Al}$ was removed using saturated calcium chloride. The AAO film was then etched in a $0.6 \mathrm{M}$ phosphoric acid solution to remove the continuous barrier layer present at the bottom of each nanopore and enlarge the nanopore diameters. Finally, an AAO film with a thickness of about $70 \mu \mathrm{m}$ and pore diameters of about $70 \mathrm{~nm}$ was obtained. Such AAO templates were used as described below, to fabricate Co nanotubes by electrochemical deposition.

$\mathrm{A} \mathrm{Cu}$ conductive layer was sputtered onto one side of the AAO template to provide an electrical contact. Electrodeposition was performed in a three-electrode cell under constant voltage at room temperature, where the sputtered conducting layer served as the working electrode. A saturated calomel electrode (SCE) was used as the reference, and a graphite pole was used as the counter electrode. The electrolyte used to prepare the Co nanotubes was prepared from reagent grade chemicals and deionized water. It consisted of $0.1 \mathrm{M} \mathrm{CoSO}_{4} \cdot 7 \mathrm{H}_{2} \mathrm{O}$ and $0.1 \mathrm{M} \mathrm{H}_{3} \mathrm{BO}_{3}$. The $\mathrm{pH}$ of the electrolyte was 2.5. The electrodeposition was carried out for $20 \mathrm{~min}$, at room temperature $\left(25^{\circ} \mathrm{C}\right)$ with different DC voltages ranging from -1.0 to $-3.0 \mathrm{~V}$. The total charge current density at any applied potential is less than $1 \mathrm{~mA} / \mathrm{cm}^{2}$. As a result, nanostructures with a length about $6 \mu \mathrm{m}$ were obtained.

The morphology of the Co nanotubes was characterized using a field-emission scanning electron microscope (SEM,
Hitachi S-4800) and a transmission electron microscope (TEM, Hitachi H-7650). The crystallographic structure and composition of the nanostructures were examined using an $\mathrm{X}$-ray diffractometer (XRD) with $\mathrm{Cu} \mathrm{K}_{\alpha}$ radiation and using X-ray energy dispersive spectroscopy (EDS). The magnetic properties were measured at room temperature using a physical properties measurement system (PPMS6700 ) with the magnetic field parallel and perpendicular to the nanostructures. Except for the TEM sample whose AAO template was completely removed by alkaline treatment, all samples used in the measurements reported here were partially embedded in the AAO.

\section{Results and discussion}

Figure 1a, $\mathrm{b}$ shows the SEM images of an AAO template before and after sputtering the $\mathrm{Cu}$ layer. We can see from Fig. 1a that the AAO pores had an average diameter of about $70 \mathrm{~nm}$. To serve efficiently as a cathode, a $\mathrm{Cu}$ layer was first sputtered onto one side of the AAO. The sputtering power and time were $2 \mathrm{~kW}$ and $20 \mathrm{~min}$, respectively. It can be seen from Fig. 1b that almost all the AAO pores were covered with $\mathrm{Cu}$. Our $\mathrm{Co}$ nanotubes and nanowires were fabricated using such an AAO template coated with $\mathrm{Cu}$. Figure 1c, d are the TEM image and EDS spectrum of a typical as-prepared nanotubes, respectively. It may be seen that the diameter of the Co nanotubes is $70 \mathrm{~nm}$ which is consistent with the AAO template structure, as shown in Fig. 1a. The EDS spectrum of the Co nanotubes confirms that the as-grown nanotubes consist of pure cobalt. The observed $\mathrm{O}, \mathrm{Al}$, and $\mathrm{C}$ peaks are generated from the alumina film and the conductive tape.

However, several groups reported that the electrodeposition product consisted of only nanowires when using an AAO template with its pores blocked [14-17]. This is inconsistent with our experiment results. We attributed it to differences in the electrodeposition parameters. The different results also imply that it is possible for a more universal growth mechanism to explain template-based electrochemical deposition of metal nanotubes. In view of this point, we have studied in detail the dependence of the Co morphology on the applied potential.

SEM images of the Co nanostructures are shown in Figs. 1c and 2a-e. The images demonstrate that our Co nanotubes have a large aspect ratio and they take on a uniform growth. The wall width of the Co nanotubes is in the range $15-18 \mathrm{~nm}$. The filling rate of the nanostructures is nearly $100 \%$. The SEM images also demonstrate that either nanowires or nanotubes can be successfully deposited by adjusting the deposition potential between -1.0 and $-3.0 \mathrm{~V}$. The figures indicate that there exists a critical potential, $v_{\mathrm{c}} \approx-1.5 \mathrm{~V}$. The deposit is in the form of a 
Fig. 1 a Front side SEM images of AAO, $\mathbf{b}$ backside SEM images of AAO with $\mathrm{Cu}$ sputtering for $20 \mathrm{~min}$, c TEM images of single Co nanotubes formed with applied potential $-1.3 \mathrm{~V}, \mathbf{d}$ EDS spectrum of a typical as-prepared nanostructure

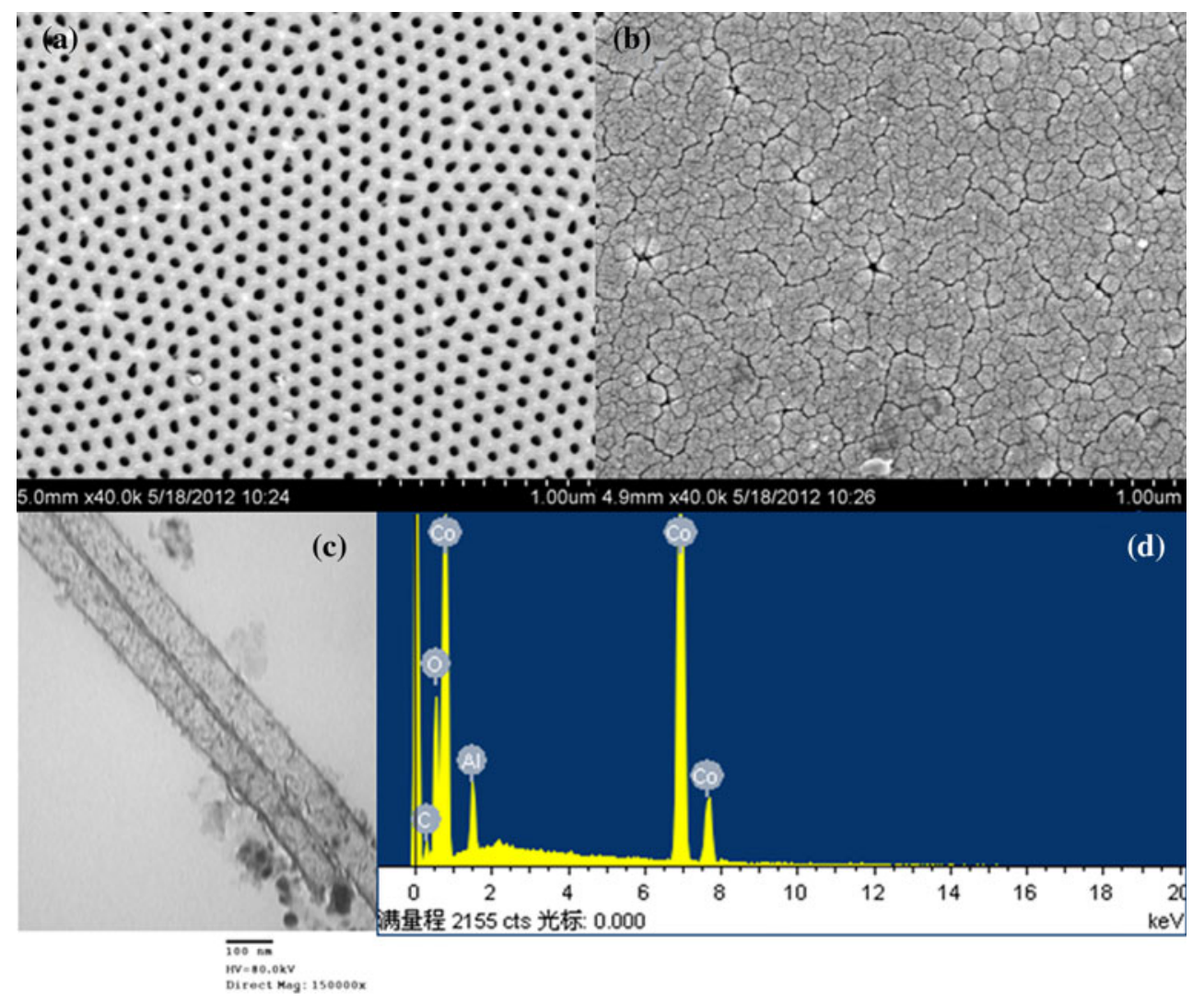

nanotube when $v \leq v_{\mathrm{c}}$. Otherwise, when $v>v_{\mathrm{c}}$, nanowires are deposited. We also found in our experiment that $v_{\mathrm{c}}$ may vary with the electrodeposition parameters (such as temperature, concentration and constituent) in a given system. It therefore appears that $v_{\mathrm{c}}$ can be defined as a critical state, below or above which the electrodeposition process is dominated by two distinct factors. According to the Reaction Principles of Physical Chemistry [18], a reaction process is determined by the two factors of kinetics and thermodynamics. Once the reaction takes place, the crystal develops in a manner determined by ambient condition. Our XRD results indicated that the Co nanotubes or nanowires all have a similar growth habit, respectively. The nanotubes are oriented growth along kinetics crystallographic plane, but the nanowires are oriented growth along thermodynamics crystallographic plane. Therefore we assumed that $v_{\mathrm{c}}$ is a critical state separating the deposition process into two sections dominated by kinetics or thermodynamics, respectively.

The XRD patterns of typical Co nanostructures (shown in Fig. 3) demonstrate that both Co nantubes and nanowires have an hcp (PDF00-001-1278) structure, but they have different crystal orientations. The sharp diffraction peaks at $75.764^{\circ}$ and $44.600^{\circ}$ correspond, respectively, to the kinetics crystallographic plane (110) and the thermodynamics crystallographic plane (002). This indicates that the kinetics crystallographic plane (110) can fully develop when $v \leq v_{\mathrm{c}}$, and that the thermodynamics crystallographic plane (002) can fully develop when $v>v_{\mathrm{c}}$. These can be attributed to the different arrangement of atom under different conditions. A foreign atom has a preferential rank among thermodynamics crystallographic planes (002) when $v>v_{\mathrm{c}}$, because the energy of the atom is so low that it has enough time to perform close packing among atoms. But when $v \leq v_{\mathrm{c}}$, the foreign atom has high energy, and the relaxation time is short. Therefore it preferentially ranks among kinetic crystallographic plane (110) which is not a closely packed crystallographic plane. Moreover, Fig. 2c-f clearly shows that the wall thickness of the Co nanotubes becomes increasingly thin as the applied negative potential decreases. This is due to the fact that the growth rate of the kinetics crystallographic plane (110) becomes much faster than that of the thermodynamics crystallographic plane (002) with an increase in the kinetics factor in electrodeposition process. These results suggest that Co nanotubes and nanowires are formed under conditions where kinetics or thermodynamics dominate the electrodeposition process, respectively. This is in good agreement with Ref. [19]. Thus, we can conclude that the electrodeposition process is dominated by kinetics or thermodynamics when $v \leq v_{\mathrm{c}}$ and $v>v_{\mathrm{c}}$, respectively.

The magnetic properties of Co nanostructures were studied at room temperature using a PPMS with the magnetic fields applied perpendicular $(\perp)$ and parallel $(\|)$ to the 

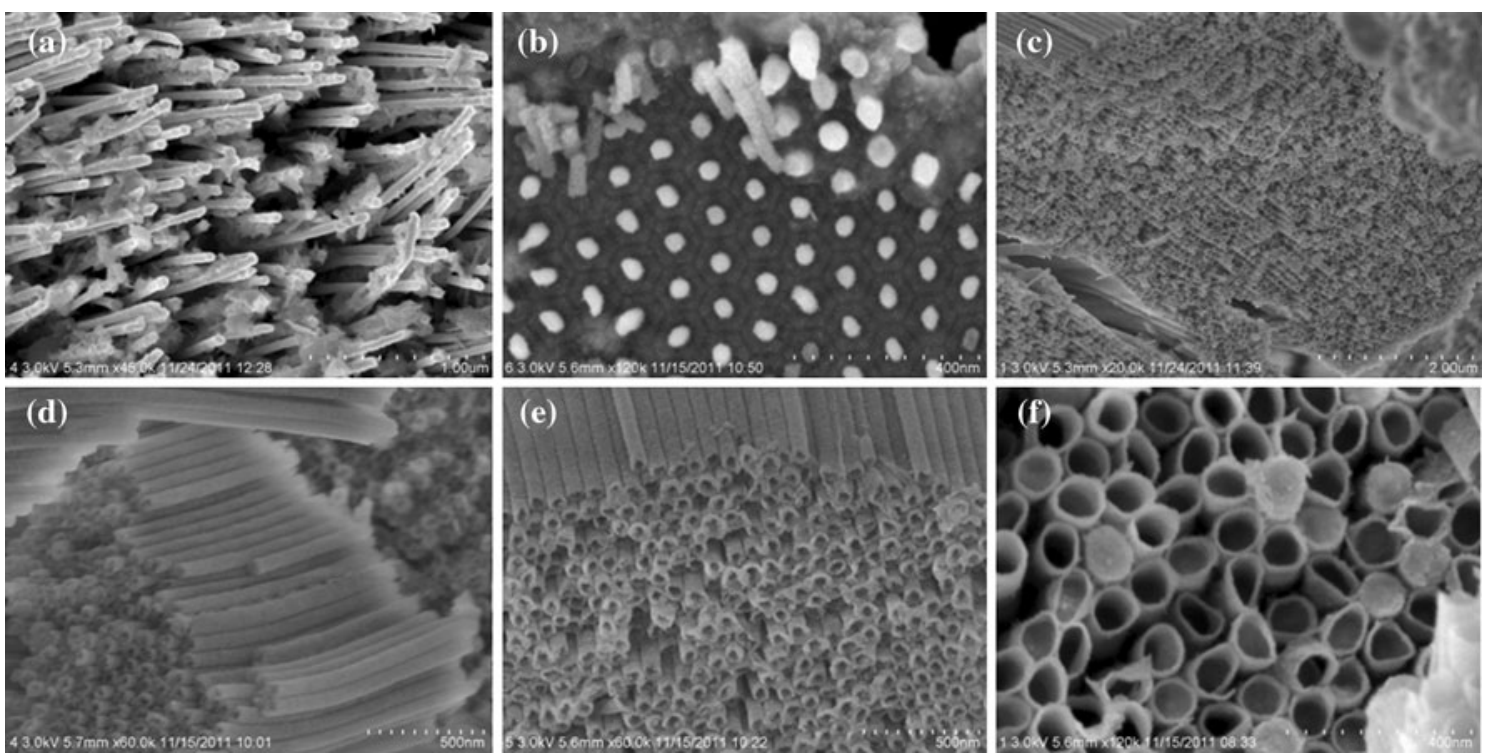

Fig. 2 SEM images of Co nanostructures: a, b nanowires formed with applied potentials of -1.0 and $-1.2 \mathrm{~V}$, respectively; c-f nanotubes formed with applied potentials of $-1.25,-1.5,-2.0$ and $-3.0 \mathrm{~V}$, respectively

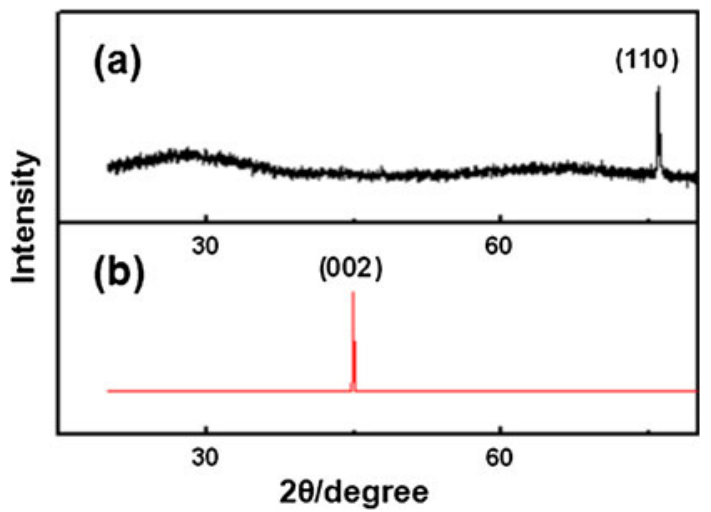

Fig. 3 XRD patterns of typical Co nanostructures, a nanotubes formed with an applied potential of $-2.0 \mathrm{~V}, \mathbf{b}$ nanowires formed with an applied potential of $-1.0 \mathrm{~V}$

long axis of the nanostructures. The magnetic parameters of the Co nanotubes with different applied potentials are shown in Table 1. These data show that the remanent squareness (SQ) and coercivities $\left(\mathrm{H}_{\mathrm{c}}\right)$ parallel to the direction of the presented nanotubes are large compared to values for the perpendicular direction, which shows that the easy axis of the Co nanotubes is parallel to the tube axis. This conclusion also follows from the magnetic hysteresis curves (shown in Fig. 4a, b), and can be attributed to the strong shape anisotropy of the nanostructures. In addition, Table 1 also shows that the value for $\mathrm{H}_{\mathrm{c}}$ first increases up to $-1.25 \mathrm{~V}$ and then decreases. We attributed the former to the structure difference between nanowires and nanotubes, and the latter to the variation of Co particles stacking density.
Table 1 Magnetic parameters, coercivity $\left(\mathrm{H}_{\mathrm{c}}\right)$ and remanent squareness (SQ) of Co nanotubes and nanowires embedded in AAO templates with the field applied parallel $(\|)$ and perpendicular $(\perp)$ to the tube long axis

\begin{tabular}{lllll}
\hline Potential $(\mathrm{V})$ & $\mathrm{SQ}^{\perp}(\mathrm{emu})$ & $\mathrm{SQ}^{\|}(\mathrm{emu})$ & $\mathrm{H}_{\mathrm{c}}^{\perp}(\mathrm{Oe})$ & $\mathrm{H}_{\mathrm{c}}^{\|}(\mathrm{Oe})$ \\
\hline-1.0 & 0.00025 & 0.001267 & 175 & 691 \\
-1.2 & 0.000276 & 0.001424 & 192 & 710 \\
-1.25 & 0.00038 & 0.00285 & 210 & 760 \\
-1.3 & 0.000394 & 0.00297 & 203 & 726 \\
-1.5 & 0.000362 & 0.00215 & 180 & 690 \\
-2.0 & 0.0006 & 0.00283 & 170 & 749 \\
-3.0 & 0.0011 & 0.0023 & 135 & 563 \\
\hline
\end{tabular}

The hysteresis loops investigated for Co nanotubes grown with different applied potentials indicated that they have similar characteristics: (1) discontinuous intervals appear in the curves, (2) the values of $\Delta \mathrm{H}$ (defined below) in each of the hysteresis loops for the nanotubes are similar. These characteristics are interesting because the hysteresis loops of Co nanowires do not have such features. The hysteresis loops of typical samples are shown in Fig. 4, where the above differences for Co nanotubes and nanowires may be clearly seen. The results imply that a reversal processes takes place in Co nanotubes that is distinct from what occurs in Co nanowire. The inset of Fig. 4d shows an enlargement of the magnetic moment dependence on the field in the discontinuous interval from $\mathrm{Hs}_{1}(1493 \mathrm{Oe})$ to $\mathrm{Hs}_{2}$ (1676 Oe). It can be seen a field range exists, $\Delta \mathrm{H}=\mathrm{H}_{\mathrm{s} 2}-\mathrm{H}_{\mathrm{s} 1}=183 \mathrm{Oe}$, over which the magnetic moment remains constant during the reversal processes. This suggests that there exists some peculiar magnetic 
Fig. 4 Hysteresis loops of Co nanostructures formed with an applied potential of $\mathbf{a}-1.0 \mathrm{~V}$ and $\mathbf{b}-2.0 \mathrm{~V}$, measured at room temperature, $\mathbf{c}$ and $\mathbf{d}$ the partial amplification of $\mathbf{a}$ and b with the applied field perpendicular to the nanostructures. Inset enlargement of $\mathbf{d}$ over the interval from 1493 to 1676 Oe
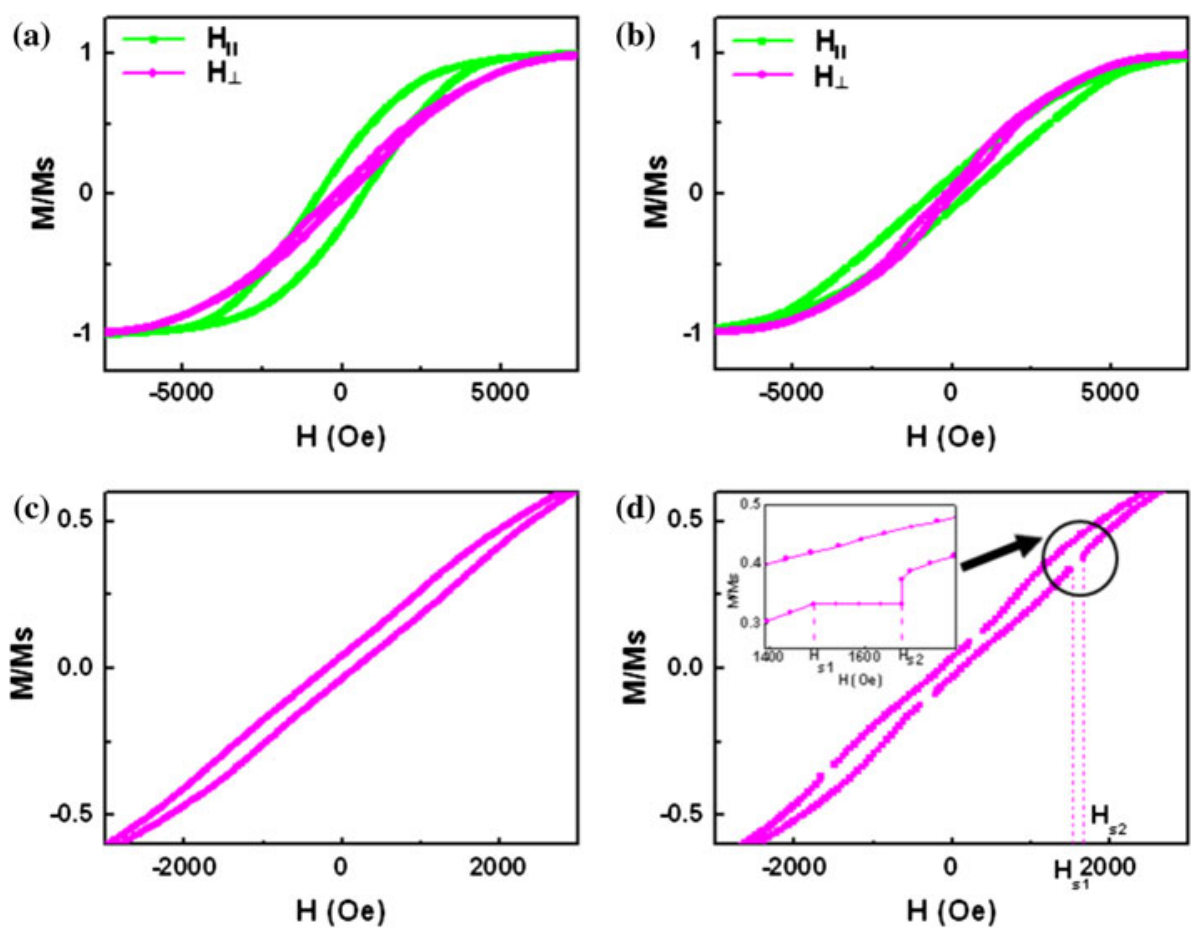

Fig. 5 Schematic diagram of vortex wall configurations in $\mathrm{Co}$ nanotubes: a $\mathrm{CW}$ alone existence, $\mathbf{b} \mathrm{CCW}$ alone existence and $\mathbf{c}$ coexistence of both $\mathrm{CW}$ and $\mathrm{CCW}$
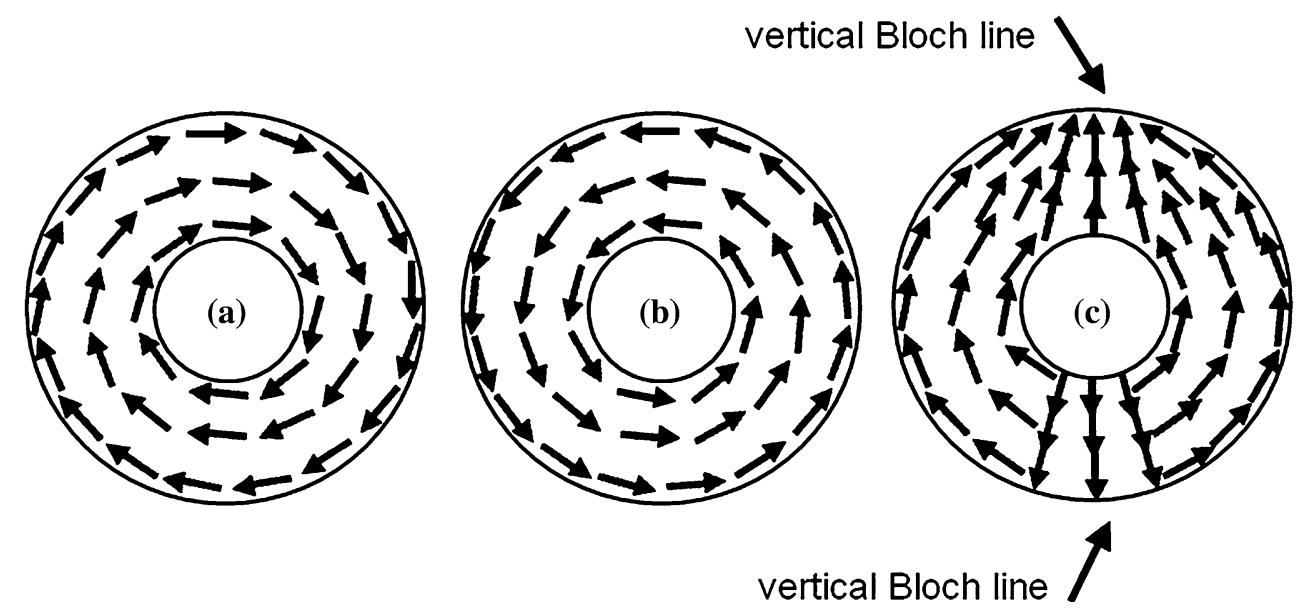

one vortex wall. In the latter situation, a peculiar configuration arises in the regions where $\mathrm{CW}$ and $\mathrm{CCW}$ vortices meet or detach. Since the configuration is similar to that of the Bloch line that has been investigated previously [2426], we refer below to this configuration as a Bloch line. With reference to magnetic bubble material, Malozemoff et al reported that the plane field needed for a single vertical Bloch line magnetization reversal is eight times of saturation moment $\left(M_{\mathrm{s}}\right)$ if the external in-plane field exerted on magnetic bubble material is parallel, but oppositely directed, to the vertical Bloch line (shown in Fig. 6) [27]. The value of $M_{\mathrm{s}}$ is given by the expression:

$M_{\mathrm{S}}=\frac{M}{\pi\left(r_{1}^{2}-r_{2}^{2}\right) h n}$ 


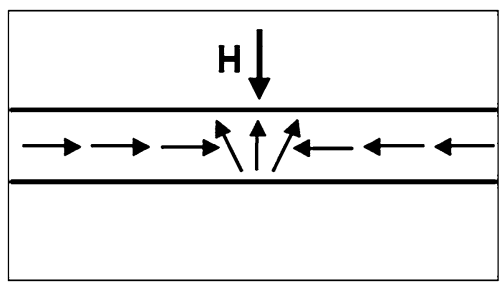

Fig. 6 Schematic diagram of the location relationship between the in-plane field and the vertical Bloch line

where $h$ and $n$ are the height and number of the nanotubes per square millimetre; $r_{1}$ and $r_{2}$ are the inner and outer radius of the nanotubes; $M_{\mathrm{s}}$ is saturation moment. We obtain the value $M_{\mathrm{s}} \approx 214 \mathrm{emu} / \mathrm{cm}^{3}$ for our Co nanotubes formed with an applied potential of $-2.0 \mathrm{~V}$. In addition, from Fig. $4 \mathrm{~d}, \quad \mathrm{H}_{\mathrm{s} 2} \approx 1676$ Oe. Therefore $8 \mathrm{M}_{\mathrm{s}}=$ $1712 \approx \mathrm{H}_{\mathrm{s} 2}$. This is in agreement with the theory of the Bloch line mentioned above. This suggests that the peculiar configuration identified above in Co nanotubes may be a vertical Bloch line (shown in Fig. 5c). Of course, the relationship between $8 \mathrm{M}_{\mathrm{s}}$ and $\mathrm{H}_{\mathrm{s} 2}$ may vary with the location of the vertical Bloch line in the Co nanotubes when the applied field is perpendicular $(\perp)$ to the long axis of the nanostructures. Nevertheless, the reversal process of these vertical Bloch lines is similar, which accounts for the discontinuous interval appearing at low applied field. During the reversal process, when $\mathrm{H}<\mathrm{H}_{\mathrm{s} 1}$ or $\mathrm{H}>\mathrm{H}_{\mathrm{s} 2}$ (Fig. $4 \mathrm{~d}$, inset), the $\mathrm{CCW}$ or $\mathrm{CW}$ vortices are immediately broken up as the external field increases, so that the hysteresis loops are continuous over these ranges. Breaking up of the vertical Bloch line is, however, very hard, and the Bloch line can remain stable in the reversal field range, $\mathrm{H}_{\mathrm{s} 1}<\mathrm{H}<\mathrm{H}_{\mathrm{s} 2}$. Once $\mathrm{H}$ increases to $\mathrm{H}_{\mathrm{s} 2}$, the vertical Bloch line instantly disappears. This give rise to the discontinuity in the hysteresis loops for the Co nanotubes.

\section{Conclusion}

Co nanotubes were fabricated using AAO templates by a DC electrodeposition method. According to the dependence of the morphology of the Co nanostructures on the applied potential, we determined a critical potential $v_{\mathrm{c}}$ for fabrication of Co nanostructures through DC electrodeposition. We defined $v_{\mathrm{c}}$ as a critical state separating the deposition process into two sections dominated by kinetics or thermodynamics, respectively. When the applied negative potential was equal to or below $v_{\mathrm{c}}$, the deposition process is dominated by kinetics. To the contrary, when the applied negative potential was above $v_{\mathrm{c}}$, the deposition process is dominated by thermodynamics. The experimental results demonstrated that Co nanotubes are the products of kinetics dominating the electrodeposition process, and that the wall thickness of the Co nanotubes became thinner as the applied potential was reduced. Magnetic measurements showed that the hysteresis loops of the Co nanotubes were discontinuous. Calculations indicate that the in-plane reversal field needed to break up the peculiar configuration responsible for the discontinuity is about eight times the saturation moment, which suggests that the existence of a vertical Bloch line is possible in $\mathrm{Co}$ nanotubes.

Acknowledgements This work is supported by the Natural Science Foundation of Hebei Province (Grant No. A2012205038).

Open Access This article is distributed under the terms of the Creative Commons Attribution License which permits any use, distribution, and reproduction in any medium, provided the original author(s) and the source are credited.

\section{References}

1. Sanchez-Castillo MA, Couto C, Kim WB, Dumesic JA (2004) Angew Chem 43:1140

2. McPhillips J, Murphy A, Jonsson MP, Hendren WR, Atkinson R, Höök F, Zayats AV, Pollard RJ (2010) ACS Nano 4:2210

3. Lee DH, Kim JE, Han TH, Hwang JW, Jeon S, Choi SY, Hong SH, Lee WJ, Ruoff RS, Kim SO (2010) Adv Mater 22:1247

4. Mor GK, Kim S, Paulose M, Varghese OK, Shankar K, Basham J, Grimes CA (2009) Nano Lett 9:4250

5. Li L, Yang YW, Huang XH, Li GH, Ang R, Zhang LD (2006) Appl Phys Lett 88:103119

6. Han XF, Shamaila S, Sharif R, Chen JY, Liu HR, Liu DP (2009) Adv Mater 21:4619

7. Li DD, Thompson RS, Bergmann G, Lu JG (2008) Adv Mater 20:4575

8. Chen JY, Liu HR, Ahmad N, Li YL, Chen ZY, Zhou WP, Han XF (2011) J Appl Phys 109:07E157

9. Ahmad N, Chen JY, Iqbal J, Wang WX, Zhou WP, Han XF (2011) J Appl Phys 109:07A331

10. Ceolin G, Orbán Á, Kocsis V, Gyurcsányi RE, Kézsmárki I, Horváth V (2013) J Mater Sci 48:5209

11. Cao HQ, Wang LD, Qiu Y, Wu QZ, Wang GZ, Zhang L, Liu XW (2006) Chem Phys Chem 7:1500

12. Nielsch K, Castano FJ, Ross CA, Krishnan R (2005) J Appl Phys 98:034318

13. Nielsch K, Castano FJ, Matthias S, Lee W, Ross CA (2005) Adv Eng Mater 7:217

14. Jie Fu, Cherevko S, Chung C-H (2008) Electrochem Commun 10:514

15. Li XR, Wang YQ, Song GJ, Peng Z, Yu YM, She XL, Sun J, Li JJ, Li PD, Wang ZF, Duan XF (2010) J Phys Chem C114:6914

16. Mingxiao Fu, Zhu Y, Tan R, Shi G (2001) Adv Mater 13:1874

17. Sun Y, Xia Y (2004) Adv Mater $16: 264$

18. Gasstone S (1946) Textbook of physical chemistry. D. Van Nostrand Company, New York

19. Zhang H, Zhang X, Zhang J, Li Z, Sun H (2013) J Electrochem Soc 160:D41

20. Landeros P, Allende S, Escrig J, Salcedo E, Altbir D (2007) Appl Phys Lett 90:102501 
21. Wang ZK, Lim HS, Liu HY, Ng SC, Kuok MH, Tay LL, Lockwood DJ, Cottam MG, Hobbs KL, Larson PR, Keay JC, Lian GD, Johnson MB (2005) Phys Rev Lett 94:137208

22. Escrig J, Landeros P, Altbir D, Vogel EE, Vargas P (2007) J Magn Magn Mater 308:233

23. Albrecht Ole, Zierold R, Allende S, Escrig J, Patzig C, Rauschenbach B, Nielsch K, Görlitz D (2011) J Appl Phys 109: 093910
24. Sun HY, Hu HN, Nie XF (2001) J Magn Magn Mater 237:169

25. Sun HY, Hu HN, Sun YP, Nie XF (2004) J Magn Magn Mater 279:241

26. Sun HY, Gu JJ, Hu HN, Jia LZ, Feng SZ, Nie XF, Sun YP (2005) J Magn Magn Mater 292:281

27. Malozemoff AP, Slonczewski JC (1979) Magnetic domain walls in bubble materials. Academic Press, New York 